\title{
Effect of fluid viscosity on wave propagation in a cylindrical bore in micropolar elastic medium
}

\author{
SUNITA DESWAL ${ }^{1}$, SUSHIL K TOMAR ${ }^{2}$ and RAJNEESH \\ KUMAR $^{3}$ \\ ${ }^{1}$ Department of Mathematics, F C College, Hisar 125001 , India \\ ${ }^{2}$ Mathematics Department, Panjab University, Chandigarh 160014 , India \\ ${ }^{3}$ Department of Mathematics, Kurukshetra University, Kurukshetra 136119 , \\ India \\ e-mail: sktomar@yahoo.com
}

MS received 29 April 1999; revised 7 September 2000

\begin{abstract}
Wave propagation in a cylindrical bore filled with viscous liquid and situated in a micropolar elastic medium of infinite extent is studied. Frequency equation for surface wave propagation near the surface of the cylindrical bore is obtained and the effect of viscosity and micropolarity on dispersion curves is observed. The earlier problems of Biot and of Banerji and Sengupta have been reduced as a special case of our problem.
\end{abstract}

Keywords. Cylindrical bore; surface wave; micropolarity; viscosity; phase velocity; dispersion equation.

\section{Introduction}

Surface wave propagation near a bore hole in an elastic host medium is of great practical importance. Since valuable organic and inorganic deposits beneath the earth's surface are difficult to detect by drilling randomly, wave propagation is the simplest and most economic technique to detect these and does not require any drilling through the earth. Almost all the oil companies rely on seismic interpretation for selecting the sites for exploratory oil wells because seismic wave methods have higher accuracy, higher resolution and are more economical, as compared to drilling which is expensive and timeconsuming.

Eringen \& Suhubi (1964) and Suhubi \& Eringen (1964) developed a nonlinear theory of micro-elastic solids. Later Eringen (1966) developed a theory for the special class of microelastic materials and called it the 'Linear theory of micropolar elasticity'. In problems of waves and vibrations, the classical theory of elasticity fails to provide complete information and the results obtained do not conform with experimental ones in case of media with granular structures. Eringen's (1966) theory of micropolar elasticity is found to be adequate in this regard, particularly in problems of waves and vibrations through granular structured media. Under this theory, solids can undergo macro deformations and 
micro-rotations. The motion in this kind of solid is completely characterized by the displacement vector $\mathbf{u}(\mathbf{x}, t)$ and the rotation vector $\boldsymbol{\phi}(\mathbf{x}, t)$, while in the case of classical elasticity, the motion is characterized by the displacement vector only. Micropolar solids can support couple stresses in addition to force stresses. Physically, solids that are composed of dumb-bell type of molecules may be adequately represented by the micropolar elasticity model. Fibrous materials and some granular and porous solids may also fall into this category (Eringen 1966). It is believed that "porous granular" material can best represent soil (Deresiewicz 1958). Therefore certain soils having nearly this composition can be approximated to the micropolar elasticity model. Motivated by this idea, we have considered the problem of propagation of surface waves in a cylindrical bore containing viscous liquid and situated in a micropolar elastic medium of infinite extent.

Nowacki (1970) and Parfitt \& Eringen (1969) have discussed the problems of propagation of waves in micropolar elastic materials. A number of problems of waves and vibrations in micropolar elastic media have been discussed by many researchers and have appeared in the open literature. The problem concerning wave propagation in cylindrical bore holes was first discussed by Biot (1952). Banerji \& Sengupta (1977) have studied the corresponding problem in micropolar elastic media. They have considered wave propagation in cylindrical empty bores with and without inviscid liquid and studied the dispersion relation, pseudo Rayleigh waves and Stoneley waves. Cheng \& Toksoz (1981) have discussed elastic wave propagation in a fluid-filled bore hole and synthetic acoustic logs. Sharma \& Gogna (1990) have studied the propagation of elastic waves in cylindrical bores in liquid-saturated porous solids of infinite extent. They have obtained the velocity dispersion equation appropriate to surface wave propagation in liquid-filled porous solids containing an empty bore; the case of liquid-filled bore is also discussed. Chi et al (1997) have considered the problem of inclined bore holes in poroelastic media. Recently, Tomar \& Kumar (1999) have investigated the problem of elastic wave propagation in cylindrical bores situated in micropolar elastic media with stretch and also the effect of micropolarity and microstretch on dispersion of surface waves in cylindrical bores with and without inviscid liquid. Here, the problem under consideration is to study the effect of micropolarity and viscosity on dispersion curves for surface waves in cylindrical bores filled with viscous liquid and situated in micropolar elastic media of infinite extent.

\section{Formulation of the problem and its solution}

We consider a circular cylindrical bore of radius $a$ in a micropolar elastic medium of infinite extent and take cylindrical polar co-ordinates $(r, \theta, z)$ with the $z$-axis pointing upward along the axis of the cylinder. Our aim is to study the propagation of axial symmetric waves which are pure sinusoidal along the axial direction. Following Eringen (1966), the equations of motion and constitutive relations for a micropolar elastic solid medium in the absence of body forces and surface couples are governed by

$$
\begin{aligned}
& (\mu+K) \nabla^{2} \mathbf{u}+(\lambda+\mu) \nabla(\nabla \cdot \mathbf{u})+K \nabla \times \boldsymbol{\phi}=\rho\left(\partial^{2} \mathbf{u} / \partial t^{2}\right), \\
& (\alpha+\beta+\gamma) \nabla(\nabla \cdot \boldsymbol{\phi})-\gamma \nabla \times(\nabla \times \boldsymbol{\phi})+K \nabla \times \mathbf{u}-2 K \boldsymbol{\phi}=\rho j\left(\partial^{2} \boldsymbol{\phi} / \partial t^{2}\right),
\end{aligned}
$$

and

$$
\begin{aligned}
t_{i j} & =\lambda \delta_{i j} u_{k, k}+\mu\left(u_{i, j}+u_{j, i}\right)+K\left(u_{j, i}-\epsilon_{i j k} \phi_{k}\right), \\
m_{j} & =\alpha \phi_{k, k} \delta_{i j}+\beta \phi_{i, j}+\gamma \phi_{j, i}
\end{aligned}
$$


where $\lambda, \mu, K, \alpha, \beta, \gamma$ are material moduli, $\rho$ is the density and $j$ is the micro-inertia of the medium considered. $t_{i j}$ and $m_{i j}$ are components of force- and couple-stress tensors respectively. $\mathbf{u}(\mathbf{x}, t)$ and $\boldsymbol{\phi}(\mathbf{x}, t)$ denote displacement and micro-rotation vectors respectively. For axial symmetric problem, we take

$$
\mathbf{u}=\left(u_{r}, 0, u_{z}\right) \text { and } \boldsymbol{\phi}=\left(0, \phi_{\theta}, 0\right),
$$

and the quantities remain independent of $\boldsymbol{\theta}$.

The above consideration would reduce (1) and (2) to the following equations,

$$
\begin{aligned}
& (\mu+K)\left(\nabla^{2}-\frac{1}{r^{2}}\right) u_{r}+(\lambda+\mu) \frac{\partial e}{\partial r}-K \frac{\partial \phi_{\theta}}{\partial z}=\rho \frac{\partial^{2} u_{r}}{\partial t^{2}} \\
& (\mu+K) \nabla^{2} u_{z}+(\lambda+\mu) \frac{\partial e}{\partial z}+\frac{K}{r} \frac{\partial\left(r \phi_{\theta}\right)}{\partial r}=\rho \frac{\partial^{2} u_{z}}{\partial t^{2}} \\
& {\left[\gamma\left(\nabla^{2}-\frac{1}{r^{2}}\right)-2 K\right] \phi_{\theta}+K\left(\frac{\partial u_{r}}{\partial z}-\frac{\partial u_{z}}{\partial r}\right)=\rho j \frac{\partial^{2} \phi_{\theta}}{\partial t^{2}}}
\end{aligned}
$$

where

$$
e=\frac{1}{r} \frac{\partial\left(r u_{r}\right)}{\partial r}+\frac{\partial u_{z}}{\partial z}, \quad \nabla^{2} \equiv \frac{\partial^{2}}{\partial r^{2}}+\frac{1}{r} \frac{\partial}{\partial r}+\frac{\partial^{2}}{\partial z^{2}} .
$$

Introducing the potentials $\phi^{\prime}, \psi$ and $\Gamma$ as follows

$$
u_{r}=\frac{\partial \phi^{\prime}}{\partial r}+\frac{\partial^{2} \psi}{\partial r \partial z}, \quad u_{z}=\frac{\partial \phi^{\prime}}{\partial z}-\left(\nabla^{2}-\frac{\partial^{2}}{\partial z^{2}}\right) \psi, \quad \phi_{\theta}=-\frac{\partial \Gamma}{\partial r},
$$

equations (5) to (7) with the help of (9) reduce to

$$
\begin{aligned}
& \square_{1} \phi^{\prime}=0, \\
& \square_{2} \psi+c_{2}^{2} \Gamma=0, \\
& \square_{3} \Gamma-\omega_{0}^{2} \nabla^{2} \psi=0,
\end{aligned}
$$

where

$$
\begin{aligned}
& \square_{1}=c_{1}^{2} \nabla^{2}-\left(\partial^{2} / \partial t^{2}\right), \quad \square_{2}=b^{2} \nabla^{2}-\left(\partial^{2} / \partial t^{2}\right), \\
& \square_{3}=c_{4}^{2} \nabla^{2}-2 \omega_{0}^{2}-\left(\partial^{2} / \partial t^{2}\right), \\
& c_{1}^{2}=(\lambda+2 \mu+K) / \rho, \quad b^{2}=(\mu+K) / \rho, \\
& c_{2}^{2}=K / \rho, \quad c_{4}^{2}=\gamma / \rho j, \quad \omega_{0}^{2}=K / \rho j .
\end{aligned}
$$

The time harmonic wave solutions of (10) to (13) are given by

$$
\begin{aligned}
\phi^{\prime} & =A_{1} K_{0}\left(\lambda_{1} r\right) \exp \{\iota(k z-\omega t)\}, \\
\psi & =\left[A_{2} K_{0}\left(\lambda_{2} r\right)+A_{2}^{\prime} K_{0}\left(\lambda_{3} r\right)\right] \exp \{\iota(k z-\omega t)\}, \\
\Gamma & =\left[A_{3} K_{0}\left(\lambda_{2} r\right)+A_{3}^{\prime} K_{0}\left(\lambda_{3} r\right)\right] \exp \{\iota(k z-\omega t)\},
\end{aligned}
$$

where

$$
\begin{aligned}
\lambda_{1}^{2} & =k^{2}-\left(\omega^{2} / c_{1}^{2}\right), \\
\lambda_{2,3}^{2} & =k^{2}-\frac{1}{2}\left[\left(\sigma_{4}^{2}+\sigma_{2}^{2}+\eta_{1}^{2}-\nu^{2}\right) \mp\left\{\left(\sigma_{4}^{2}-\sigma_{2}^{2}-\nu^{2}+\eta_{1}^{2}\right)^{2}+4 \eta_{1}^{2} \sigma_{2}^{2}\right\}^{1 / 2}\right], \\
\sigma_{2}^{2} & =\omega^{2} / b^{2}, \quad \sigma_{4}^{2}=\omega^{2} / c_{4}^{2}, \quad \eta_{1}^{2}=K^{2} / \gamma(\mu+K), \quad \nu^{2}=2 K / \gamma,
\end{aligned}
$$


and $K_{0}()$ is the modified Bessel function of order zero and second kind, $\omega(=k c)$ is angular frequency, $k$ is the wave number, $c$ is the phase velocity and $A_{i}(i=1,2,3)$ and $A_{j}^{\prime}(j=2,3)$ are unknowns. Substituting (15) and (16) into (11) and (12), we obtain

$$
A_{3}=b_{2} A_{2}, \quad A_{3}^{\prime}=b_{3} A_{2}^{\prime},
$$

where

$$
b_{j}=\left(k^{2}-\sigma_{2}^{2}-\lambda_{j}^{2}\right) / p, \quad(j=2,3), \quad p=K /(\mu+K) .
$$

In order to obtain the equation of motion in a viscous liquid medium, the elastic wave equation is modified by replacing Lame's parameter $\lambda$ by $K^{\prime}-(2 / 3) \mu^{\prime}$, where $K^{\prime}$ is the bulk modulus and $\mu^{\prime}$ is the rigidity of the fluid considered. Using the correspondence principle (Ewing et al 1957) the effect of fluid viscosity is accounted for on replacing $\mu^{\prime}$ by the operator $\eta(\partial / \partial t)$, where $\eta$ is the fluid viscosity. Thus the equations of motion for wave propagation in a viscous liquid are

$$
\begin{aligned}
& \left(K^{\prime}+\frac{4}{3} \eta \frac{\partial}{\partial t}\right) \nabla^{2} \phi^{*}=\rho^{\prime} \frac{\partial^{2} \phi^{*}}{\partial t^{2}}, \\
& \eta \frac{\partial}{\partial t}\left(\nabla^{2} \psi_{i}^{*}\right)=\rho^{\prime} \frac{\partial^{2} \psi_{i}^{*}}{\partial t^{2}},
\end{aligned}
$$

where $\rho^{\prime}$ is the fluid density. Here the equations are written in terms of the scalar potential $\phi^{*}$, and vector potential $\psi$. The components of displacement are found from

$$
\mathbf{U}=\nabla \phi^{*}+\nabla \times \psi^{*}
$$

Keeping in view the above mentioned modifications, the expressions for stresses in viscous liquid media are given by

$$
\tau_{i j}=\left(K^{\prime}-\frac{2}{3} \eta \frac{\partial}{\partial t}\right) U_{k, k} \delta_{i j}+\eta \frac{\partial}{\partial t}\left(U_{i, j}+U_{j, i}\right)
$$

Since the problem is axisymmetric, we take $\mathbf{U}=\left(U_{r}, 0, U_{z}\right)$, and obtain from (21), the requisite components of displacement as

$$
U_{r}=\frac{\partial \phi^{*}}{\partial r}+\frac{\partial^{2} \psi^{*}}{\partial r \partial z}, \quad U_{z}=\frac{\partial \phi^{*}}{\partial z}-\left(\nabla^{2}-\frac{\partial^{2}}{\partial z^{2}}\right) \psi^{*} .
$$

The time-harmonic wave solutions of (19) and (20) may be written as (for $c<c_{1}$ )

$$
\begin{aligned}
\phi^{*} & =A_{4} I_{0}(r \zeta) \exp \{\iota(k z-\omega t)\}, \\
\psi^{*} & =A_{5} I_{0}\left(r \zeta^{\prime}\right) \exp \{\iota(k z-\omega t)\},
\end{aligned}
$$

where

$$
\begin{aligned}
\zeta & =\left[k^{2}-\left(\omega^{2} / c_{1}^{2}\right)\right]^{1 / 2}, \quad \zeta^{\prime}=\left[k^{2}-\left(\iota \omega / c_{l_{0}}^{2}\right)\right]^{1 / 2}, \\
c_{l}^{2} & =\left(K^{\prime}-(4 / 3) \iota \omega \eta\right) / \rho^{\prime}, \quad c_{l_{0}}^{2}=\eta / \rho^{\prime}
\end{aligned}
$$

and $I_{0}(\quad)$ is the modified Bessel function of order zero and first kind. Other symbols have their usual meanings and have been defined earlier. 


\section{Boundary conditions}

Boundary conditions are the continuity of stresses and displacements at the interface $r=a$, between the micropolar elastic medium and the viscous liquid. Since viscous liquid cannot support couple stresses (or micro-rotation), the stresses must therefore vanish there. Mathematically, these boundary conditions can be expressed as: At $r=a$

$$
t_{r r}=\tau_{r r}, \quad t_{r z}=\tau_{r z}, \quad m_{r \theta}=0, \quad u_{r}=U_{r}, \quad u_{z}=U_{z} .
$$

Making use of (3), (4), (9), (14)-(17) and (22)-(26) in the above boundary conditions (27), we obtain a system of five homogeneous equations in five unknowns namely $A_{1}, A_{2}, A_{2}^{\prime}, A_{4}$ and $A_{5}$. Elimination of these unknowns would give us the frequency equation

$$
\left|a_{i j}\right|=0 .
$$

The non-zero elements of (28) are written in non-dimensional form as below.

$$
\begin{aligned}
a_{11}= & {\left[\frac{\lambda_{1}^{2} a^{2}}{k^{2} a^{2}}+\frac{\lambda}{2 \mu+K}\left(\frac{\lambda_{1}^{2} a^{2}}{k^{2} a^{2}}-1\right)\right] \frac{K_{0}\left(\lambda_{1} a\right)}{K_{1}\left(\lambda_{1} a\right)}+\frac{\lambda_{1}^{2} a^{2}}{k^{2} a^{2}} \frac{1}{\lambda_{1} a}, } \\
a_{1 i}= & \frac{\lambda_{i}^{2} a^{2}}{k^{2} a^{2}}\left[\frac{K_{0}\left(\lambda_{i} a\right)}{K_{1}\left(\lambda_{i} a\right)}+\frac{1}{\lambda_{i} a}\right], \\
a_{14}= & \frac{2 \eta \omega}{2 \mu+K} \frac{y_{2}-\iota x_{2}}{k a}-\left[\frac{K^{\prime}}{2 \mu+K}\left(x_{2}^{2}-y_{2}^{2}-1+2 \iota x_{2} y_{2}\right)\right. \\
& \left.+\frac{8}{3} \frac{\eta \omega}{2 \mu+K}\left(x_{2} y_{2}+\frac{\iota}{2}\left(x_{2}^{2}-y_{2}^{2}+\frac{1}{2}\right)\right)\right] \frac{I_{0}(\zeta a)}{I_{1}(\zeta a)}, \\
a_{15}= & \frac{2 \eta \omega}{2 \mu+K}\left[\frac{x_{1}+\iota y_{1}}{k a}-\frac{I_{0}\left(\zeta^{\prime} a\right)}{I_{1}\left(\zeta^{\prime} a\right)}\left(x_{1}^{2}-y_{1}^{2}+2 \iota x_{1} y_{1}\right)\right], \\
a_{21}= & \frac{\lambda_{1} a}{k a}, \quad a_{2 i}=\left[1+\frac{K}{2 \mu+K} \frac{b_{i}}{k^{2}}+\frac{\mu+K}{2 \mu+K}\left(\frac{\lambda_{i}^{2} a^{2}}{k^{2} a^{2}}-1\right)\right] \frac{\lambda_{i} a}{k a}, \\
a_{24}= & \frac{2 \eta \omega}{2 \mu+K}\left(y_{2}-\iota x_{2}\right), \quad a_{25}=\frac{\eta \omega}{2 \mu+K}\left[x_{1}\left(x_{1}^{2}-3 y_{1}^{2}+1\right)+\iota y_{1}\left(3 x_{1}^{2}-y_{1}^{2}+1\right)\right], \\
a_{3 i}= & \frac{\lambda_{i}^{2} a^{2}}{k^{2} a^{2}}\left[\frac{1}{\lambda_{i} a}+\frac{\gamma}{\beta+\gamma} \frac{K_{0}\left(\lambda_{i} a\right)}{K_{1}\left(\lambda_{i} a\right)}\right] \frac{b_{i}}{k^{2}}, \quad a_{41}=\frac{\lambda_{1} a}{k a}, \quad a_{4 i}=\frac{\lambda_{i} a}{k a}, \\
a_{44}= & x_{2}+\iota y_{2}, \quad a_{45}=-\left[y_{1}-\iota x_{1}\right], \quad a_{51}=\frac{K_{0}\left(\lambda_{1} a\right)}{K_{1}\left(\lambda_{1} a\right)}, \\
a_{5 i}= & \frac{\lambda_{i}^{2} a^{2}}{k^{2} a^{2}} \frac{K_{0}\left(\lambda_{i} a\right)}{K_{1}\left(\lambda_{i} a\right)}, \quad a_{54}=-\frac{I_{0}(\zeta a)}{I_{1}(\zeta a)}, \\
a_{55}= & {\left[2 x_{1} y_{1}+\iota\left(y_{1}^{2}-x_{1}^{2}\right)\right] \frac{I_{0}\left(\zeta^{\prime} a\right)}{I_{1}\left(\zeta^{\prime} a\right)}, \quad i=2,3, }
\end{aligned}
$$

where

$$
x_{1}=\frac{1}{\sqrt{2}}\left\{1+\left(1+\frac{c^{4}}{c_{l_{0}}^{4} \omega^{2}}\right)\right\}^{1 / 2}, \quad y_{1}=\frac{1}{\sqrt{2}}\left\{\left(1+\frac{c^{4}}{c_{l_{0}}^{4} \omega^{2}}\right)-1\right\}^{1 / 2},
$$




$$
\begin{aligned}
& x_{2}=\frac{1}{\sqrt{2}}\left[1-\frac{c^{2} \rho^{\prime} K^{\prime}}{K^{\prime 2}+\frac{16}{9} \omega^{2} \eta^{2}}+\left(1+\frac{c^{2} \rho^{\prime}\left(c^{2} \rho^{\prime}-2 K^{\prime}\right)}{K^{\prime 2}+\frac{16}{9} \omega^{2} \eta^{2}}\right)^{1 / 2}\right]^{1 / 2}, \\
& y_{2}=\frac{1}{\sqrt{2}}\left[-1+\frac{c^{2} \rho^{\prime} K^{\prime}}{K^{\prime 2}+\frac{16}{9} \omega^{2} \eta^{2}}+\left(1+\frac{c^{2} \rho^{\prime}\left(c^{2} \rho^{\prime}-2 K^{\prime}\right)}{K^{\prime 2}+\frac{16}{9} \omega^{2} \eta^{2}}\right)^{1 / 2}\right]^{1 / 2} .
\end{aligned}
$$

\section{Special cases}

(I) In order to reduce the problem of wave propagation in a cylindrical bore filled with homogeneous, inviscid liquid and passing through a micropolar elastic medium of infinite extent, we make the viscosity of the fluid $n$ equal to zero. In this case the frequency equation (28) reduces to

$$
\begin{aligned}
& a_{1}\left[a_{2} b_{3} \lambda_{3}\left\{\frac{\beta+\gamma}{\lambda_{3} a}+\gamma \frac{K_{0}\left(\lambda_{3} a\right)}{K_{1}\left(\lambda_{3} a\right)}\right\}-a_{3} b_{2} \lambda_{2}\left\{\frac{\beta+\gamma}{\lambda_{2} a}+\gamma \frac{K_{0}\left(\lambda_{2} a\right)}{K_{1}\left(\lambda_{2} a\right)}\right\}\right] \\
& -(2 \mu+K)^{2} k^{2} \lambda_{1} \lambda_{2} \lambda_{3}\left[\left(\frac{1}{\lambda_{2} a}+\frac{K_{0}\left(\lambda_{2} a\right)}{K_{1}\left(\lambda_{2} a\right)}\right)\left(\frac{\beta+\gamma}{\lambda_{3} a}+\gamma \frac{K_{0}\left(\lambda_{3} a\right)}{K_{1}\left(\lambda_{3} a\right)}\right) b_{3}\right. \\
& \left.-\left(\frac{1}{\lambda_{3} a}+\frac{K_{0}\left(\lambda_{3} a\right)}{K_{1}\left(\lambda_{3} a\right)}\right)\left(\frac{\beta+\gamma}{\lambda_{2} a}+\gamma \frac{K_{0}\left(\lambda_{2} a\right)}{K_{1}\left(\lambda_{2} a\right)}\right) b_{2}\right] \\
& =M \lambda_{1}\left[b_{3} \lambda_{3}\left(\frac{\beta+\gamma}{\lambda_{3} a}+\gamma \frac{K_{0}\left(\lambda_{3} a\right)}{K_{1}\left(\lambda_{3} a\right)}\right)\left\{a_{2}-(2 \mu+K) k^{2}\right\}\right. \\
& \left.-b_{2} \lambda_{2}\left(\frac{\beta+\gamma}{\lambda_{2} a}+\gamma \frac{K_{0}\left(\lambda_{2} a\right)}{K_{1}\left(\lambda_{2} a\right)}\right)\left\{a_{3}-(2 \mu+K) k^{2}\right\}\right]
\end{aligned}
$$

where

$$
\begin{aligned}
& M=\frac{\rho^{\prime} \omega^{2}}{\zeta_{0}} \frac{I_{0}\left(\zeta_{0} a\right)}{I_{1}\left(\zeta_{0} a\right)}, \quad \zeta_{0}^{2}=k^{2}\left(1-\frac{c^{2}}{y^{2}}\right), \\
& y^{2}=\frac{K^{\prime}}{\rho^{\prime}} \quad a_{1}=(2 \mu+K) \lambda_{1}^{2}\left[\frac{1}{\lambda_{1} a}+\frac{K_{0}\left(\lambda_{1} a\right)}{K_{1}\left(\lambda_{1} a\right)}\right], \\
& a_{i}=(2 \mu+K) k^{2}+K b_{i}+(\mu+K)\left(\lambda_{i}^{2}-k^{2}\right), \quad i=2,3,
\end{aligned}
$$

and $b_{j}$ 's are defined earlier. The dispersion equation (31) coincides with the dispersion equation obtained by Banerji \& Sengupta (1977) for the relevant problem.

(II) If we neglect the viscous effect of the liquid along with the micropolar effect of the medium, then the problem reduces to that of wave propagation in a cylindrical bore filled with inviscid liquid and situated in a classical elastic medium. For this case, we use $\eta=0$ and $K=\beta=\gamma=0$ in the frequency equation (28), and obtain

$$
\begin{gathered}
{\left[2 \mu \sigma^{2}\left\{\frac{1}{\sigma a}+\frac{K_{0}(\sigma a)}{K_{1}(\sigma a)}\right\}+\lambda\left(\sigma^{2}-k^{2}\right) \frac{K_{0}(\sigma a)}{K_{1}(\sigma a)}\right]\left(k^{2}+\lambda_{2}^{\prime 2}\right)} \\
-4 \mu k^{2} \sigma \lambda_{2}^{\prime}\left\{\frac{1}{\lambda_{2}^{\prime} a}+\frac{K_{0}\left(\lambda_{2}^{\prime} a\right)}{K_{1}\left(\lambda_{2}^{\prime} a\right)}\right\}=\frac{\omega^{2} \rho^{\prime}}{\zeta_{0}} \frac{I_{0}\left(\zeta_{0} a\right)}{I_{1}\left(\zeta_{0} a\right)} \sigma\left(\lambda_{2}^{\prime 2}-k^{2}\right),
\end{gathered}
$$


where

$$
\begin{aligned}
& \sigma^{2}=k^{2}-\frac{\omega^{2}}{c_{1}^{2}}, \quad c_{1}^{2}=(\lambda+2 \mu) / \rho, \quad \lambda_{2}^{\prime 2}=k^{2}-\frac{\omega^{2}}{b_{2}^{2}}, \\
& b_{2}^{2}=\frac{\mu}{\rho}, \quad \zeta_{0}^{2}=k^{2}-\frac{\omega^{2}}{c_{f}^{2}}, \quad c_{f}^{2}=K^{\prime} / \rho^{\prime} .
\end{aligned}
$$

Equation (33) corresponds to the dispersion equation obtained by Biot (1952) for the relevant problem.

(III) If we neglect the micropolar effect of the medium by putting $K=\beta=\gamma=0$ in the frequency equation (28), then we obtain the frequency equation for waves propagating in the bore hole filled with viscous liquid and situated in an elastic medium. The reduced frequency equation is given by,

$$
\left|d_{i j}\right|=0,
$$

where the elements of this determinantal equation are given by

$$
\begin{aligned}
d_{11}= & {\left[\frac{\sigma^{2} a^{2}}{k^{2} a^{2}}+\frac{\lambda}{2 \mu}\left(\frac{\sigma^{2} a^{2}}{k^{2} a^{2}}-1\right)\right] \frac{K_{0}(\sigma a)}{K_{1}(\sigma a)}+\frac{\sigma^{2} a^{2}}{k^{2} a^{2}} \frac{1}{\sigma a}, } \\
d_{12}= & \frac{\lambda_{2}^{\prime} a^{2}}{k^{2} a^{2}}\left[\frac{K_{0}\left(\lambda_{2}^{\prime} a\right)}{K_{1}\left(\lambda_{2}^{\prime} a\right)}+\frac{1}{\lambda_{2}^{\prime} a}\right], \\
d_{13}= & \frac{\eta \omega}{\mu} \frac{y_{2}-\iota x_{2}}{k a}-\left[\frac{K^{\prime}}{2 \mu}\left(x_{2}^{2}-y_{2}^{2}-1+2 \iota x_{2} y_{2}\right)\right. \\
& \left.+\frac{8}{3} \frac{\eta \omega}{2 \mu}\left(x_{2} y_{2}+\frac{\iota}{2}\left(x_{2}^{2}-y_{2}^{2}+\frac{1}{2}\right)\right)\right] \frac{I_{0}(\zeta a)}{I_{1}(\zeta a)}, \\
d_{14}= & \frac{\eta \omega}{\mu}\left[\frac{x_{1}+\iota y_{1}}{k a}-\frac{I_{0}\left(\zeta^{\prime} a\right)}{I_{1}\left(\zeta^{\prime} a\right)}\left(x_{1}^{2}-y_{1}^{2}+2 \iota x_{1} y_{1}\right)\right], \\
d_{21}= & \frac{\sigma a}{k a}, \quad d_{22}=\left[1+\frac{1}{2}\left(\frac{\lambda_{2}^{2} a^{2}}{k^{2} a^{2}}-1\right)\right] \frac{\lambda_{2} a}{k a}, \\
d_{23}= & \frac{\eta \omega}{\mu}\left(y_{2}-\iota x_{2}\right), \quad d_{24}=\frac{\eta \omega}{2 \mu}\left[x_{1}\left(x_{1}^{2}-3 y_{1}^{2}+1\right)+\iota y_{1}\left(3 x_{1}^{2}-y_{1}^{2}+1\right)\right], \\
d_{31}= & \frac{\sigma a}{k a}, \quad d_{32}=\frac{\lambda_{2} a}{k a}, \quad d_{33}=x_{2}+\iota y_{2}, \\
d_{34}= & -\left[y_{1}-\iota x_{1}\right], \quad d_{41}=\frac{K_{0}(\sigma a)}{K_{1}(\sigma a)}, \quad d_{42}=\frac{\lambda_{2}^{2} a^{2}}{k^{2} a^{2}} \frac{K_{0}\left(\lambda_{2} a\right)}{K_{1}\left(\lambda_{2} a\right)}, \\
d_{43}= & -\frac{I_{0}(\zeta a)}{I_{1}(\zeta a)}, \quad d_{44}=\left[2 x_{1} y_{1}+\iota\left(y_{1}^{2}-x_{1}^{2}\right)\right] \frac{I_{0}\left(\zeta^{\prime} a\right)}{I_{1}\left(\zeta^{\prime} a\right)},
\end{aligned}
$$

where symbols are as defined earlier.

\section{Numerical results and discussion}

In order to study numerically the effect of viscosity and micropolarity on dispersion curves, we have solved the frequency equation (28) for phase velocity by taking different values of 
wave number in non-dimensional form. In fact, the numerical results could have been more informative if the values of elastic constants appearing in the text for soil were known. Values of micropolar elastic constants for soil (a granular porous medium) were not available readily in the existing literature. To understand the problem in greater detail numerically, we decided to take the values of relevant elastic parameters for the following two materials.

Material 1: Aluminium-epoxy matrix (Gautheir 1982, p. 459):

$$
\begin{aligned}
\lambda & =7.59 \times 10^{10} \text { dynes } / \mathrm{cm}^{2}, \quad \mu=1.89 \times 10^{10} \text { dynes } / \mathrm{cm}^{2}, \\
K & =0.0149 \times 10^{10} \text { dynes } / \mathrm{cm}^{2}, \quad \beta=2.26 \times 10^{8} \text { dynes }, \\
\gamma & =2.63 \times 10^{8} \text { dynes }, \quad j=0.196 \times 10^{-2} \mathrm{~cm}^{2}, \\
\rho & =2.192 \mathrm{gm} / \mathrm{cm}^{3}, \quad \omega^{2} / \omega_{0}^{2}=8.0 .
\end{aligned}
$$

Material 2: Magnesium crystal-like material (Eringen 1984)

$$
\begin{aligned}
& \lambda=9.4 \times 10^{11} \text { dynes } / \mathrm{cm}^{2}, \quad \mu=4.0 \times 10^{11} \text { dynes } / \mathrm{cm}^{2}, \\
& K=1.0 \times 10^{11} \text { dynes } / \mathrm{cm}^{2}, \quad \beta=0.669 \times 10^{-4} \text { dynes }, \\
& \gamma=0.779 \times 10^{-4} \text { dynes }, \quad j=0.2 \times 10^{-15} \mathrm{~cm}^{2}, \\
& \rho=1.74 \mathrm{gm} / \mathrm{cm}^{3}, \quad \omega^{2} / \omega_{0}^{2}=8.0 .
\end{aligned}
$$

In order to observe clearly the effect of viscosity, we have considered kerosene oil and castor oil as low and high viscosity fluids respectively. The elastic parameters for these fluids are taken as (Lynch 1980, pp 363, 366),

for kerosene oil, a low viscosity fluid:

$$
\begin{aligned}
c_{l} & =1320 \times 10^{2} \mathrm{~cm} / \mathrm{s}, \quad \rho^{\prime}=0.8201 \mathrm{gm} / \mathrm{cm}^{3}, \\
\eta & =0.0164 \text { poise }
\end{aligned}
$$

for castor oil, a high viscosity fluid:

$$
\begin{aligned}
c_{l} & =1474 \times 10^{2} \mathrm{~cm} / \mathrm{s}, \quad \rho^{\prime}=0.9561 \mathrm{gm} / \mathrm{cm}^{3}, \\
\eta & =6.50 \text { poise. }
\end{aligned}
$$

The values of dimensionless phase velocity $c_{0}\left(=c / c_{1}\right)$ are obtained as a function of dimensionless wave number $k a$. Figures $1-4$ show the effect of micropolarity on dispersion curves for the two fluids considered. It can be seen that the effect of micropolarity is significant only for small values of $k a$ when material 1 is used, while it is significant for all values of $k a$ when material 2 is used. For instance, when the bore is filled with kerosene oil, it can be seen from figure 2 that in the range $0.0<k a<6.0$, the dispersion curves for surface waves in the bore hole through a micropolar elastic medium shown by the solid curve is more than the dispersion for the surface waves in the bore hole through an elastic medium shown by the dashed curve. But for higher values of $k a$; i.e. when $k a>6.0$ the two curves overlap showing that the effect of micropolarity is absent. Similarly, when the bore is filled with castor oil, we see from figure 1 that the effect of micropolarity on dispersion curves prevails in the range $0<k a<20$, while for values of $k a$ higher than 20 , the effect is not appreciable. In figure 4 , the effect of micropolarity cannot be observed in the range 


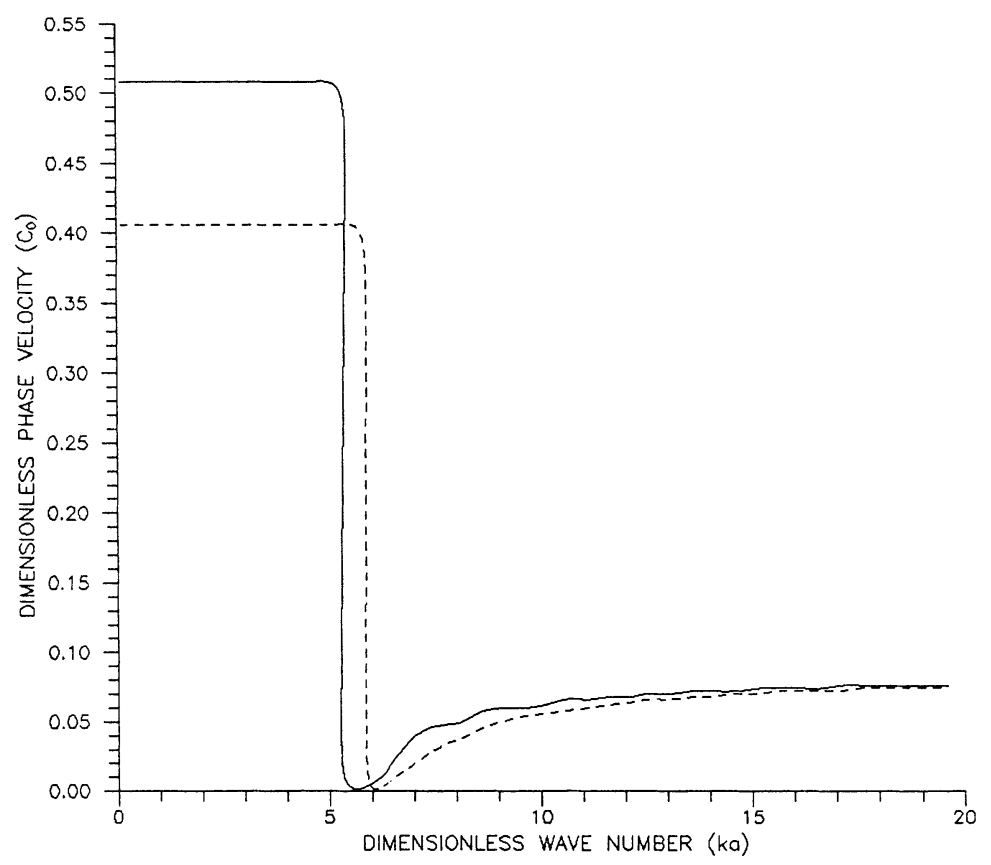

Figure 1. (Material 1) Effect of micropolarity on dispersion curves when bore is filled with castor oil. (Solid curve - when bore hole is in micropolar solid. Dashed curve - when bore hole is in elastic solid.)

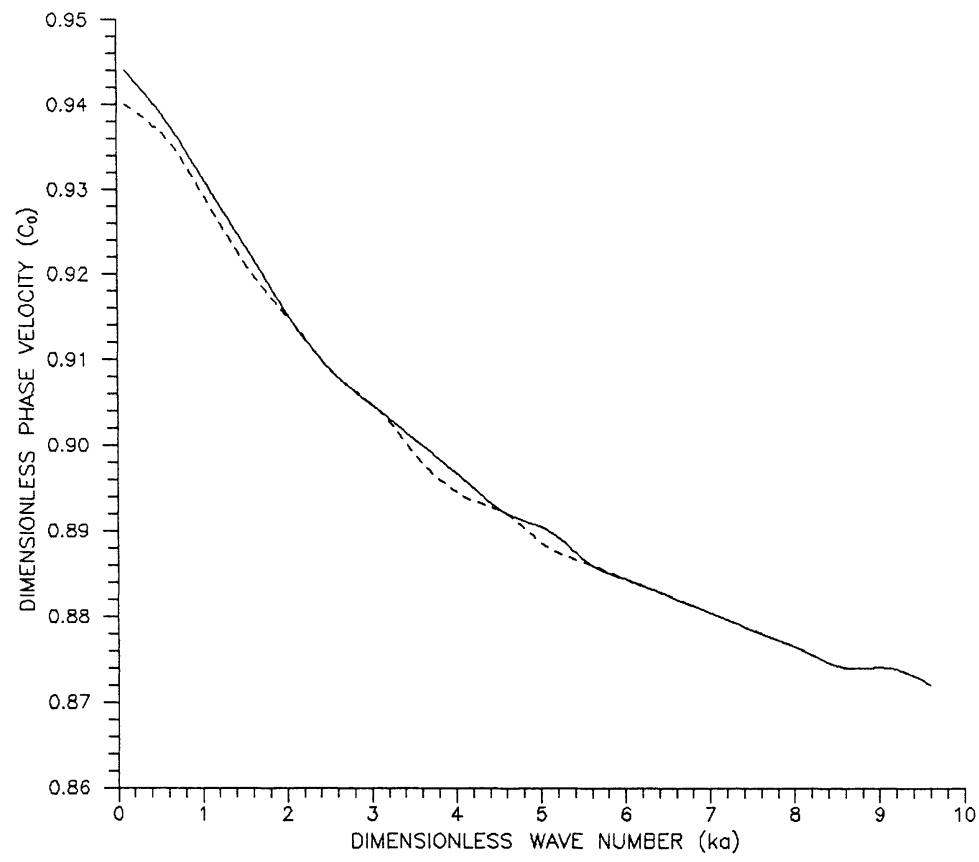

Figure 2. (Material 1) Effect of micropolarity on dispersion curves when bore is filled with kerosene oil. (Solid curve - when bore hole is in micropolar solid. Dashed curve - when bore hole is in elastic solid.) 


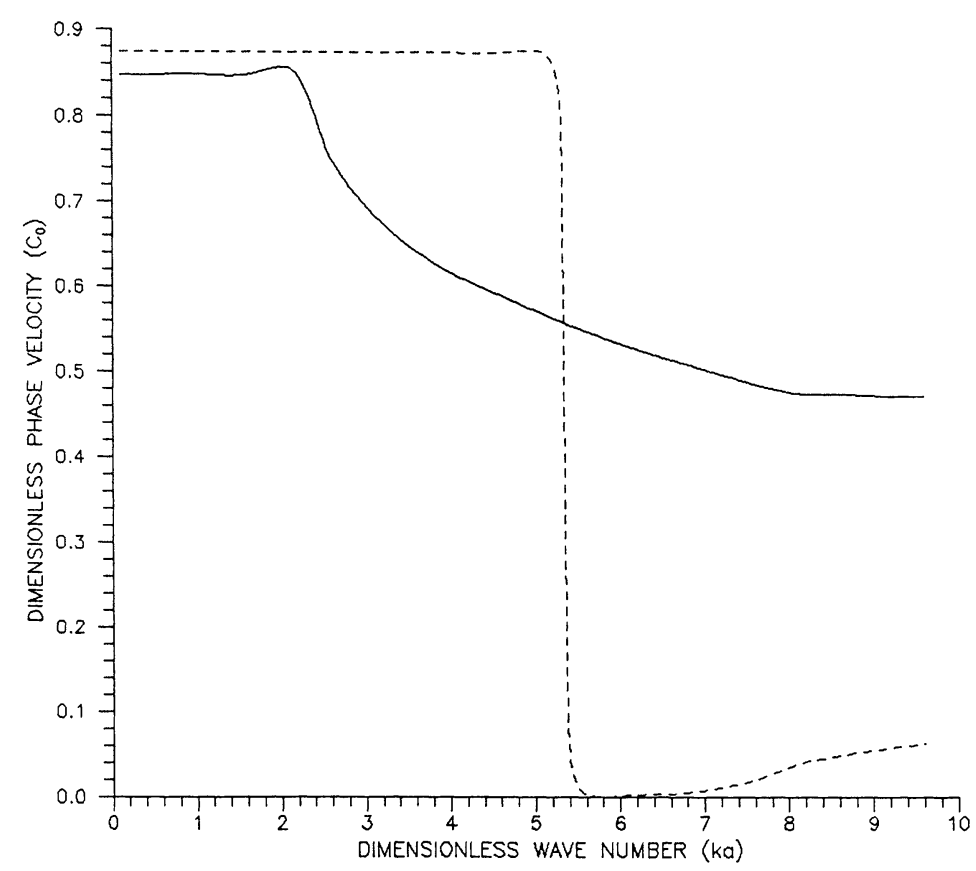

Figure 3. (Material 2) Effect of micropolarity on dispersion curves when castor oil is filled in the bore hole. (Solid curve - when bore hole is in micropolar solid. Dashed curve - when bore hole is in elastic solid.)

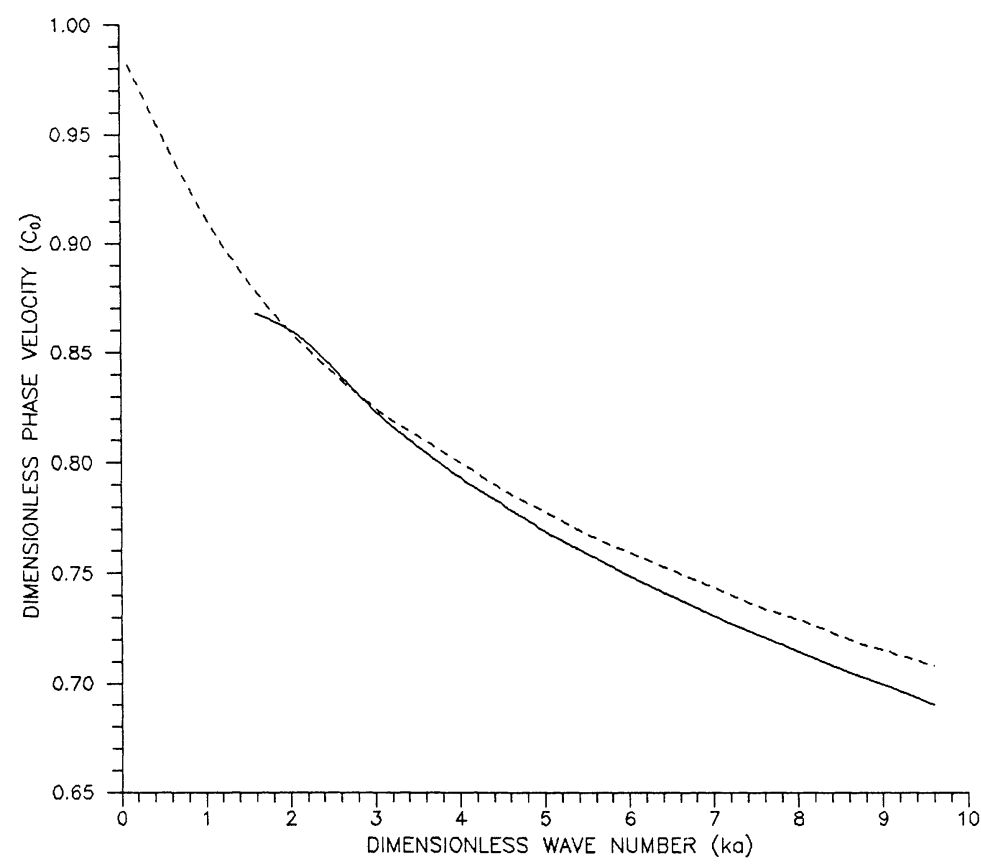

Figure 4. (Material 2) Effect of micropolarity on dispersion curves when kerosene oil is filled in the bore hole. (Solid curve - when bore hole is in micropolar solid. Dashed curve - when bore hole is in elastic solid.) 
$0<k a<1.5$ as the values of phase velocity for surface waves in a bore filled with kerosene oil could not be calculated. Figures 5-8 show the effect of viscosity on the dispersion curves. It can be seen from figures 5 and 7 that there is a clear and significant effect of viscosity on the dispersion curves. The solid curve corresponds to the dispersion curve for the surface waves when the bore is filled with castor oil and the dashed curve corresponds to the dispersion curve for the surface waves when the bore is filled with inviscid liquid. When the bore is filled with kerosene oil, we can see (figures 6 and 8), the effect of viscosity on the dispersion curves, though it is not appreciable. In figure 6 , the first three modes of propagation have been shown represented by curves I, II and III. Curve IV represents the mode of wave propagation when the bore is filled with inviscid liquid. Comparison of dispersion curves III and IV makes it clear that the viscosity of the liquid has an effect on the dispersion curve but the effect is small. The same observation can be also made from figure 8 .

\section{Conclusions}

A mathematical study is presented here to determine the effect of micropolarity and viscosity on surface wave dispersion in bore holes. The problem is reduced to that of (i) wave propagation in bore holes situated in micropolar media and filled with inviscid liquids, (ii) wave propagation in cylindrical bore holes in elastic media and filled with inviscid liquid, and (iii) wave propagation in cylindrical bore holes situated in elastic media and filled with viscous fluids. The frequency equation for each problem is obtained from

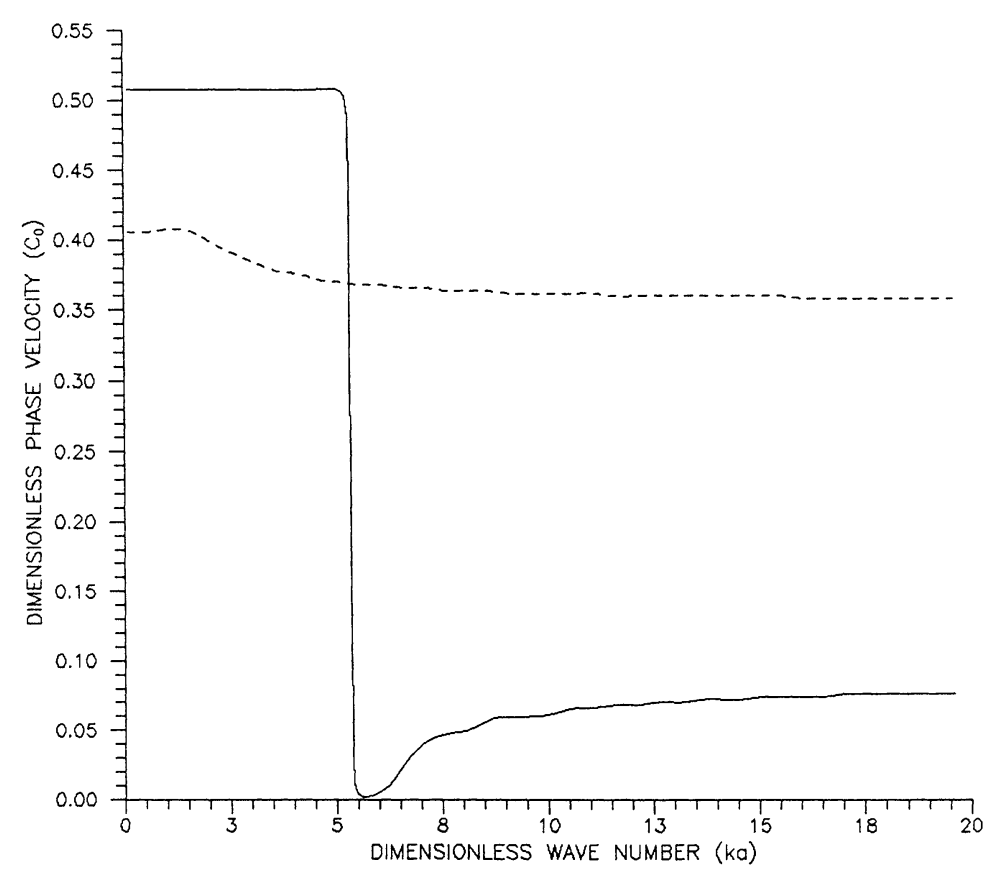

Figure 5. (Material 1) Effect of liquid viscosity on dispersion curves (Solid curve - when bore is filled with castor oil. Dashed curve - when bore is filled with inviscid liquid.) 


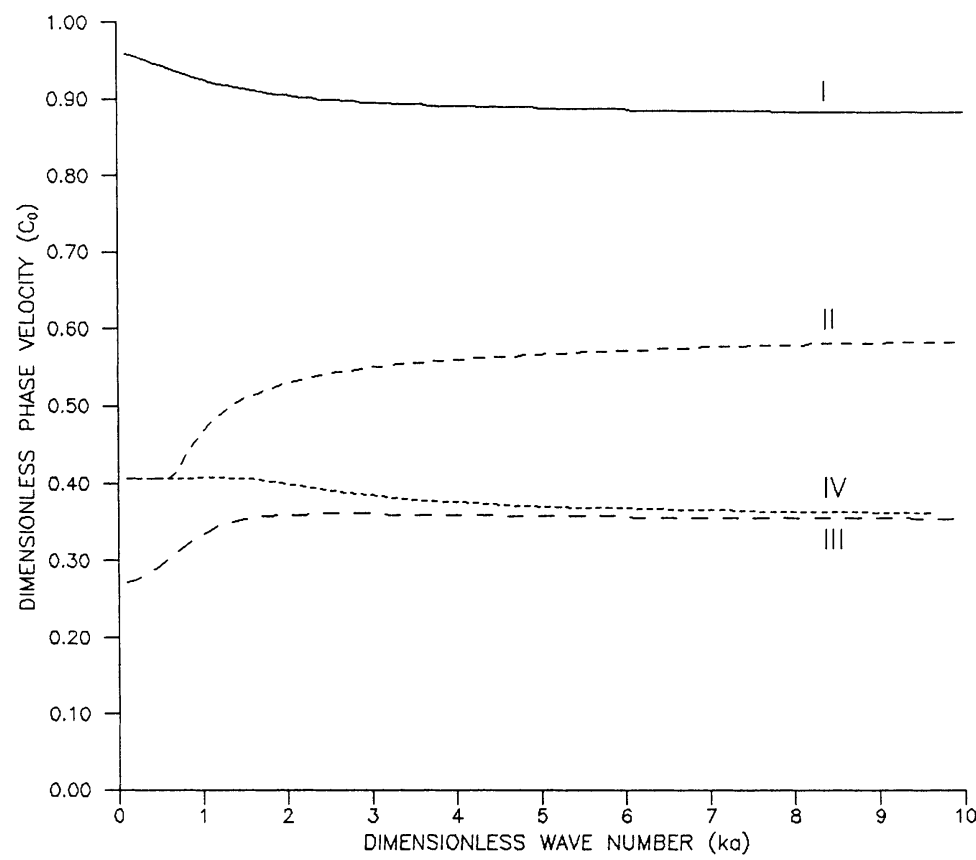

Figure 6. (Material 1) Effect of liquid viscosity on dispersion curves. (Curves I, II, III - when bore is filled with kerosene oil; Curve IV - when bore is filled with inviscid liquid.)

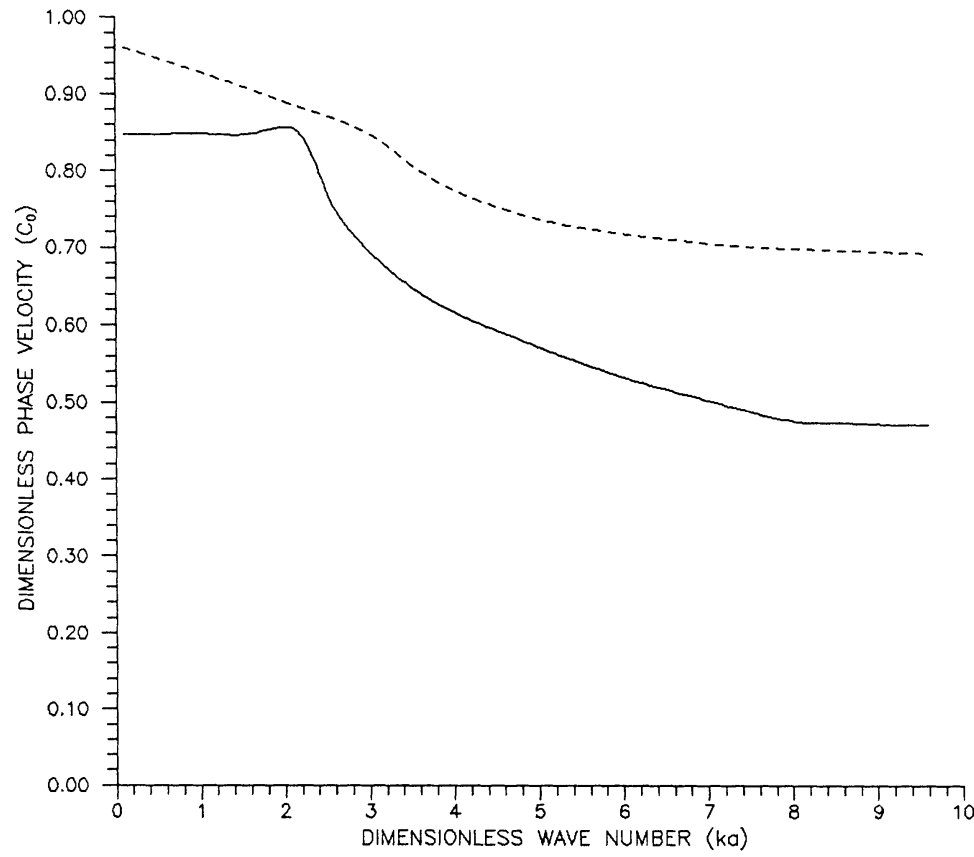

Figure 7. (Material 2) Effect of viscosity on dispersion curves. (Solid curve- when bore is filled with castor oil. Dashed curve - when bore is filled with inviscid liquid.) 


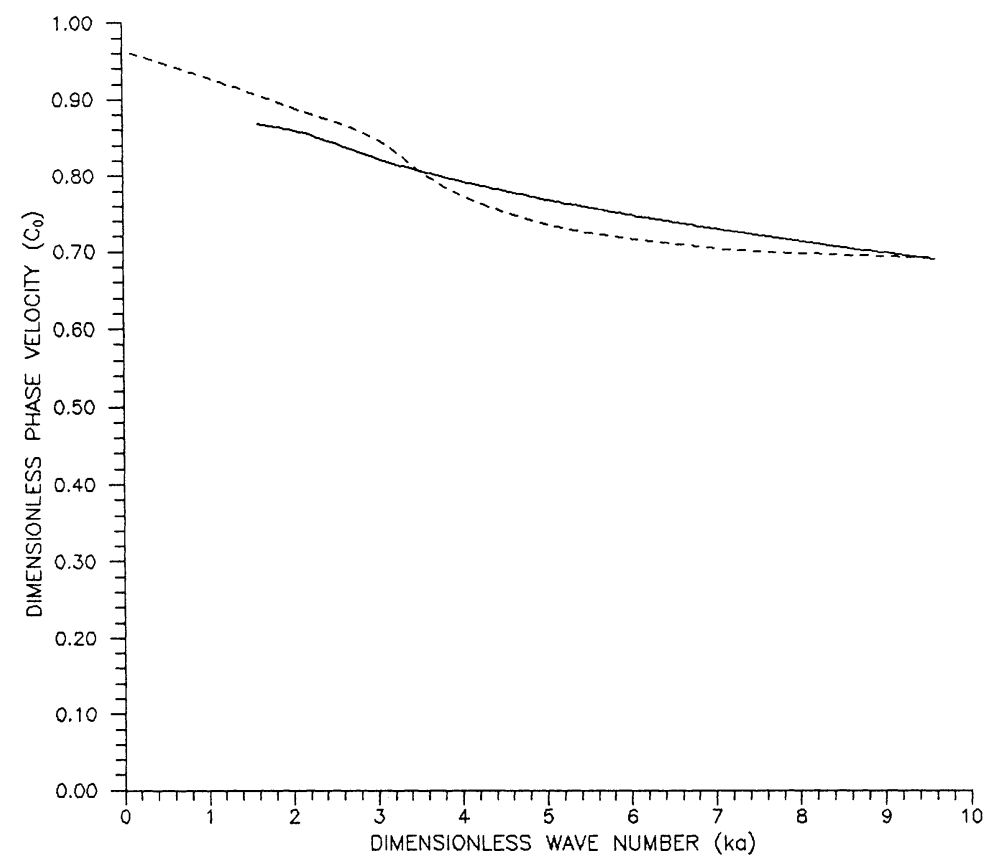

Figure 8. (Material 2) Effect of viscosity on dispersion curves. (Solid curve - when bore is filled with kerosene oil. Dashed curve - when bore is filled with inviscid liquid.)

the frequency equation of the present problem. Numerical computations are performed to solve the frequency equations and the following inferences are made.

(i) It is seen that phase velocity of wave propagation depends on wave number, showing that the frequency equation is dispersive.

(ii) The effect of micropolarity is significant for small values of $k a$ while for higher values, there is no effect of micropolarity when material 1 is used. However, it is observed for all positive values of $k a$ when material 2 is used.

(iii) Very little effect of viscosity is found on the dispersion curve for low viscosity fluids, while for highly viscous fluids a strong effect is observed. For highly viscous liquids, the effect of viscosity on dispersion curves is seen for all values of $k a>0$.

(iv) Phase velocity of mode of propagation in the bore, when filled with kerosene oil, remains in the neighbourhood of the phase velocity of the mode of propagation in the bore when filled with inviscid liquid. For material 1 , the difference between the dispersion curves for kerosene and inviscid liquid is somewhat appreciable in the range $0<k a<5$, while for $k a>5$, these two curves overlap. This means that kerosene oil, which is a low viscosity fluid behaves like an inviscid liquid. The other two modes of propagation (curves I and II), when the bore is filled with kerosene oil has higher values and differ from the mode of propagation in the case of bore filled with inviscid liquid.

Similarly, for material 2 , this effect is seen in the range $1.5<k a<9.5$ and for higher values of $k a$ it is seen that kerosene oil behaves like an inviscid fluid.

The authors are grateful to the referees for suggesting improvements in the paper. 


\section{References}

Biot M A 1952 Propagation of elastic waves in a cylindrical bore containing a fluid. J. Appl. Phys. 23: 997-1005

Banerji D K, Sengupta P R 1977a Micropolar elastic waves in a cylindrical bore containing a fluid-I. Bull. Acad. Polon. Sci. Ser. Sci. Technol. 25: 257

Banerji D K, Sengupta P R 1977b Micropolar elastic waves in a cylindrical bore containing a fluidII. Bull. Acad. Polon. Sci. Ser. Sci. Technol. 25: 263

Cheng C H, Toksoz M N 1981 Elastic wave propagation in a fluid filled bore hole and synthetic acoustic logs. Geophysics 46: 1052-1053

Chi L, Cheng A H-D, Abousleiman Y 1997 Poroelastic solution for an inclined bore hole. J. Appl. Mech. 64: 32-38

Deresiewicz H 1958 Mechanics of granular matter. In Advances in applied mathematics (eds) H L Dryden, T H von Karman (New York: Academic Press) 5: 233-303

Eringen A C 1966 Linear theory of micropolar elasticity. J. Math. Mech. 15: 909-923

Eringen A C 1984 Plane waves in nonlocal micropolar elasticity. Int. J. Eng. Sci. 22: 1113-1121

Eringen A C, Suhubi E S 1964 Nonlinear theory of simple micro-elastic solids I. Int. J. Eng. Sci. 2: 189-203

Ewing W M, Jardetzky W S, Press F 1957 Elastic waves in layered media (New York: Mc-Graw Hill)

Gautheir R D 1982 Mechanics of micropolar media (eds) O Brulin, R K T Hsieh (Singapore: World Scientific)

Lynch C T 1980 Handbook of material science. Vol. I, General properties (Boca Raton, FL: CRC Press)

Nowacki W 1970 Theory of micropolar elasticity (Courses and Lectures, International Centre for Mechanical Sciences) (Udine: Springer)

Parfitt V R, Eringen A C 1969 Reflection of plane waves from the flat boundary of a micropolar elastic half-space. J. Acoust. Soc. Am. 45: 1258-1272

Sharma M D, Gogna M L 1990 Propagation of elastic waves in a cylindrical bore in a liquid saturated porous solid. Geophys. J. Int. 103: 47-54

Suhubi E S, Eringen A C 1964 Nonlinear theory of micro-elastic solids II. Int. J. Eng. Sci. 2: 389404

Tomar S K, Kumar R 1999 Elastic wave propagation in a cylindrical bore situated in a micropolar elastic medium with stretch. Proc. Indian Acad. Sci. (Math. Sci.) 109: 425-433 